caregivers' perspectives on food-related interventions. This paper reports on a qualitative study to investigate informal caregivers' experiences of, and views on, food - related interventions in dementia. Twenty informal caregivers were interviewed and the transcripts from these interviews were analysed using both inductive and deductive thematic analysis. Three categories emerged. 'Direct food-related interventions', covers written material, training and lunch clubs 'Indirect non-food related interventions' covers respite services and domestic help at home. Finally 'No interventions' covers those who did not feel they needed any form of intervention due to confidence in managing food-related processes or having no change in dementia progression and food responsibility. Most caregivers will need different levels of education and support at different stages of dementia. It is necessary therefore to undertake ongoing individual assessment of food education and support needs. This study is the first to explore dementia and food-related social support and education needs and availability from the perspective of caregivers. A further, longitudinal study could investigate further the extent to which food-related interventional needs change over time. As dementia increases, caregivers seek more education and social support, therefore the timing of receiving this intervention is important to prepare caregivers in advance and to prevent negative outcomes occurring later.

\title{
011 PP FOOD-RELATED INTERVENTIONS IN DEMENTIA: A QUALITATIVE STUDY OF CAREGIVERS' PERSPECTIVES
}

\author{
I Papachristou, ${ }^{1 *} \mathrm{G}$ Hickey, ${ }^{2} \mathrm{~S}$ |llife'. ' University College of London, UK; \\ ${ }^{2}$ St George's University of London, UK \\ *Presenting author.
}

\subsection{6/bmjopen-2015-UCLSymposiumAbstracts.22}

As dementia progresses, caregivers increasingly have to manage the decline of food-related abilities with little intervention. The provision of food coping skills and knowledge can lessen the burden on caregivers. However, there is little research on 